\title{
Craniopharyngioma: A Case Report of Ectopic Recurrence
}

\author{
Xia Dong JIAN ${ }^{1}$, David SHRESTHA ${ }^{1}$, Zhang ZHUANG ${ }^{2}$ \\ ${ }^{1}$ First Affiliated Hospital of Liaoning Medical University, Department of Neurosurgery, Jinzhou, Liaoning 121001, China \\ ${ }^{2}$ First affiliated Hospital of Liaoning Medical University, Department of Neurology, Jinzhou, Liaoning 121001, China
}

\section{ABSTRACT}

Ectopic recurrence of craniopharyngioma is extremely rare. Two mechanisms have been proposed to explain this phenomenon: direct implantation along the previous surgical tract and dissemination via cerebrospinal fluid (CSF). We present a case of ectopic recurrence along the surgical route after transcranial gross total resection (GTR) of primary tumor. Cystic tumor seems to be a risk factor of ectopic recurrence and one must be extra careful when manipulating such tumors. We emphasize GTR and the importance of complete aspiration of cystic fluid before resecting the capsule to avoid rupture or spillage. Long-term clinical and radiological follow-up is recommended as ectopic recurrence may occur extremely late.

KEYWORDS: Craniopharyngioma, Ectopic recurrence, Cystic tumor

\section{INTRODUCTION}

$\longrightarrow$ raniopharyngioma is the rare intracranial tumor with an incidence of $0.13 / 10000$ person-years (2).

It represents $2 \%-3 \%$ of all intracranial tumors and $6 \%-13 \%$ of childhood brain tumors (5). Originating from squamous epithelium remnants of Rathke's pouch, it mostly involves the sellar/parasellar region. Craniopharyngiomas are classified as World Health Organization (WHO) tumor grade I (8) which is histologically benign with very few exceptions $(1,4,11)$. Gross total resection (GTR) is the goal of treatment. However, when GTR is not achievable, limited or subtotal resection with radiation therapy can be applied considering the relatively higher incidence of recurrence (12). Recurrence mostly occurs in the original location but exceptional cases of ectopic recurrence cannot be ignored. Two mechanisms have been raised to explain this phenomenon: direct implantation along the surgical tract during the procedure and seeding via cerebrospinal fluid (CSF).

\section{CASE REPORT}

A 42-year-old male patient was treated in our hospital 5 years ago for headache and decrease in visual acuity. He was diagnosed with craniopharyngioma (Figure 1A,B) and GTR was performed via a right pterional approach. Histopathological examination reported the papillary type(Figure 2). After surgery, the vision was normal and no radiotherapy was suggested. The patient was in regular follow-up for 3 years and then irregularly afterward. Recently the patient was admitted to our department for severe headache and dizziness for 2 months. Routine magnetic resonance imaging (MRI) revealed a cystic mass of $5 \mathrm{~cm}^{\star} 4 \mathrm{~cm}$ located in the right frontal lobe (Figure 1C). Neurological examination was intact. The right frontolateral approach was used and a cystic mass containing yellowish thin fluid was revealed while opening the dura. The lesion was totally removed (Figure 1D) and craniopharyngioma of papillary type was confirmed (Figure 3A). Ki-67 proliferation index of the recurrent tumor was 3\% (Figure 3B). Post-operative course was uneventful. No radiotherapy was prescribed. Patient was symptom-free without neurological defect.

\section{DISCUSSION}

Craniopharyngioma represents benign histology but tends to behave aggressively. It infiltrates nearby tissues and adheres to surrounding vital structures including the hypothalamus, major vessels and optic pathway. These features make total removal of the tumor challenging. GTR is the better option 

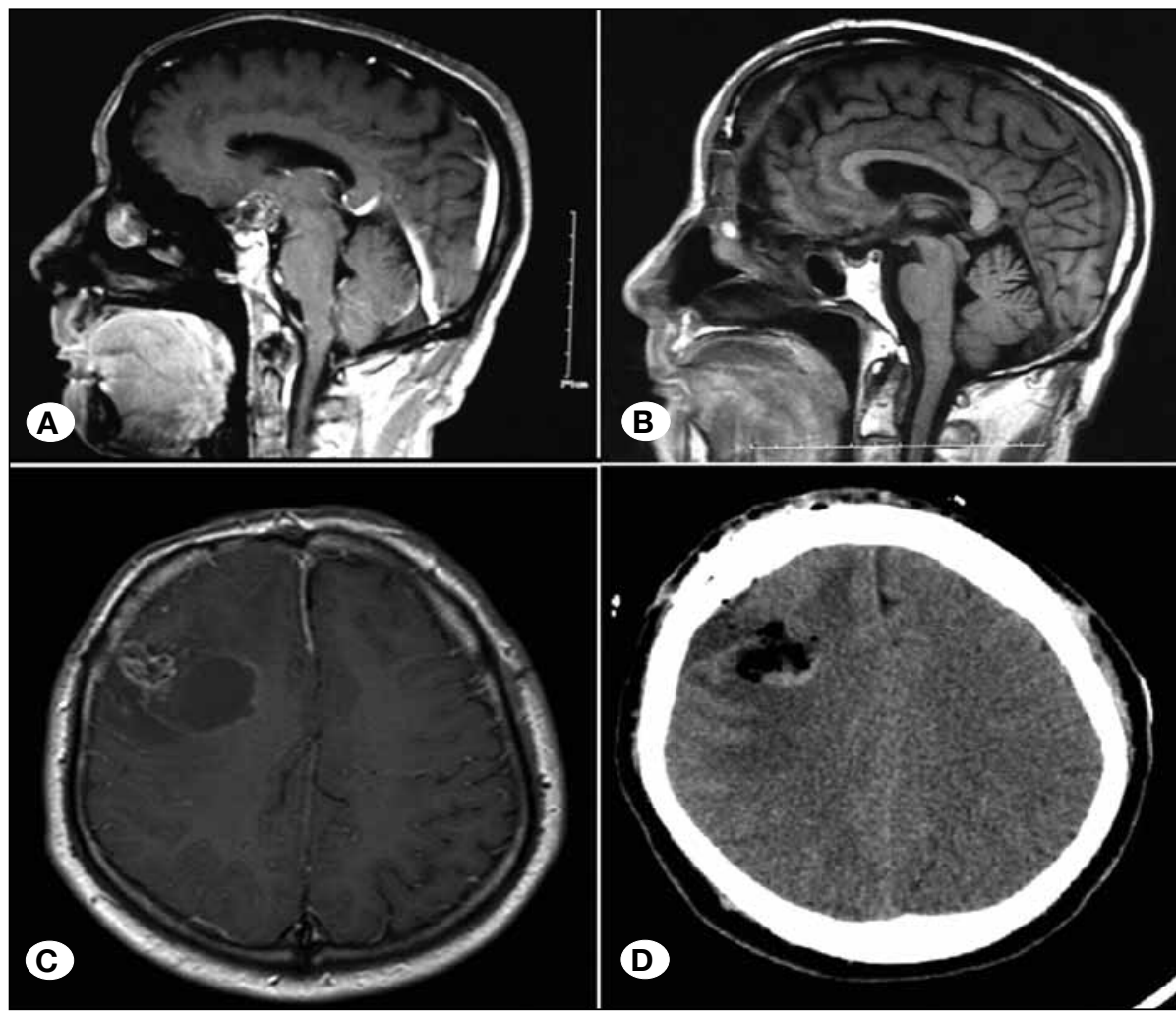

Figure 1: A) T1-weighted enhanced sagittal MRI demonstrating cystic tumor in situ; B) Postoperative T1-weighted enhanced sagittal MRI demonstrating no residual tumor; C) T1-weighted axial MRI demonstrating cystic lesion in the right frontal lobe; D) Postoperative axial Computed Tomography (CT).

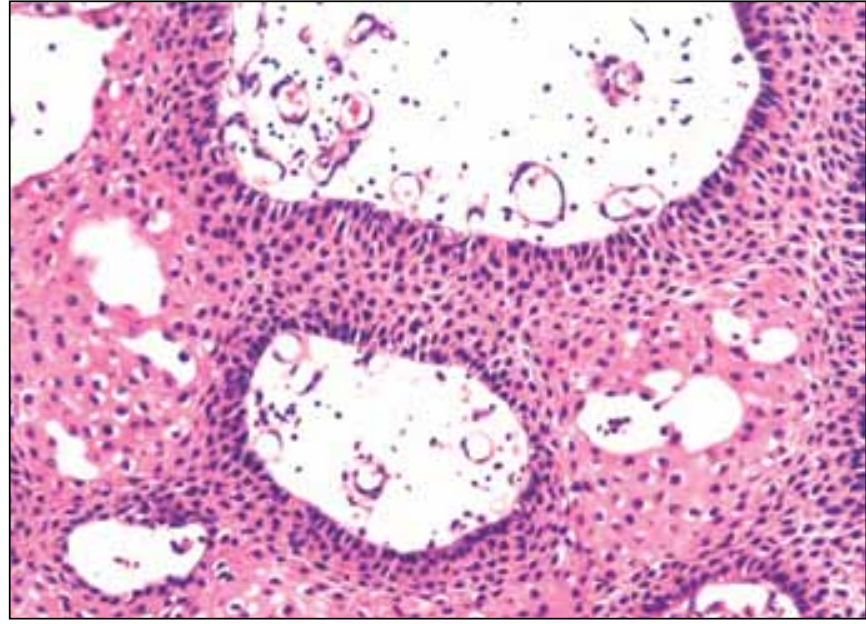

Figure 2: Hematoxylin\&Eosin staining of primary tumor confirm papillary craniopharyngioma.

with long-term tumor control and less recurrence $(3,9)$ but followed by substantial mortality. The incidence of ectopic recurrence is hard to determine because only a few cases have been reported in large studies. So far no case of spontaneous metastatic craniopharyngioma has been reported. All cases were followed by surgical intervention.

Though various authors propose several surgical techniques to prevent surgical seeding, these techniques seem to be favorable only occasionally. We concluded one interesting observation during literature review; all reported ectopic tumors are partially or totally cystic including the papillary type and the same feature was confirmed in primary tumors whose information was available (7). The high incidence of cystic component in primary tumors may be associated with ectopic recurrence after previous surgery (6).

There are two presumed mechanisms to explain the present phenomenon based on the relationship between the recurrence site and previous surgical area. The more common one appears to be direct implantation of neoplastic cells or miniature tumor tissue along the surgical route which represent the majority of all reported cases. The less frequent one is spread of tumor cells via contaminated CSF flow to a remote place (7). It is suspected that seeding via CSF may play a more important role than it appears because the site of seeding along CSF flow could involve the previous surgical route. Nomura et al. (10) were able to confirm tumor cell clusters in the CSF following the operation and two separate ectopic lesions were attributed to both mechanisms in their case. One possible explanation is that cystic tumors are prone to rupture while manipulating and the spillage of cystic fluid with tumor cells would cause contamination of surgical area or CSF. In our case, we found the tumor was in the previous surgical tract and we believe CSF contamination with cystic tumor cells only along the surgical tract is the main cause of recurrence. Preoperative and postoperative CSF analysis for cytology was negative. Patient was in regular follow up for the first three years after primary surgery and multiple scans were performed. During those periods not even a single sign or symptom of recurrence was encountered. Hence, we conclude that direct implantation is less probable in this case. Further studies on the pathogenesis of tumor 


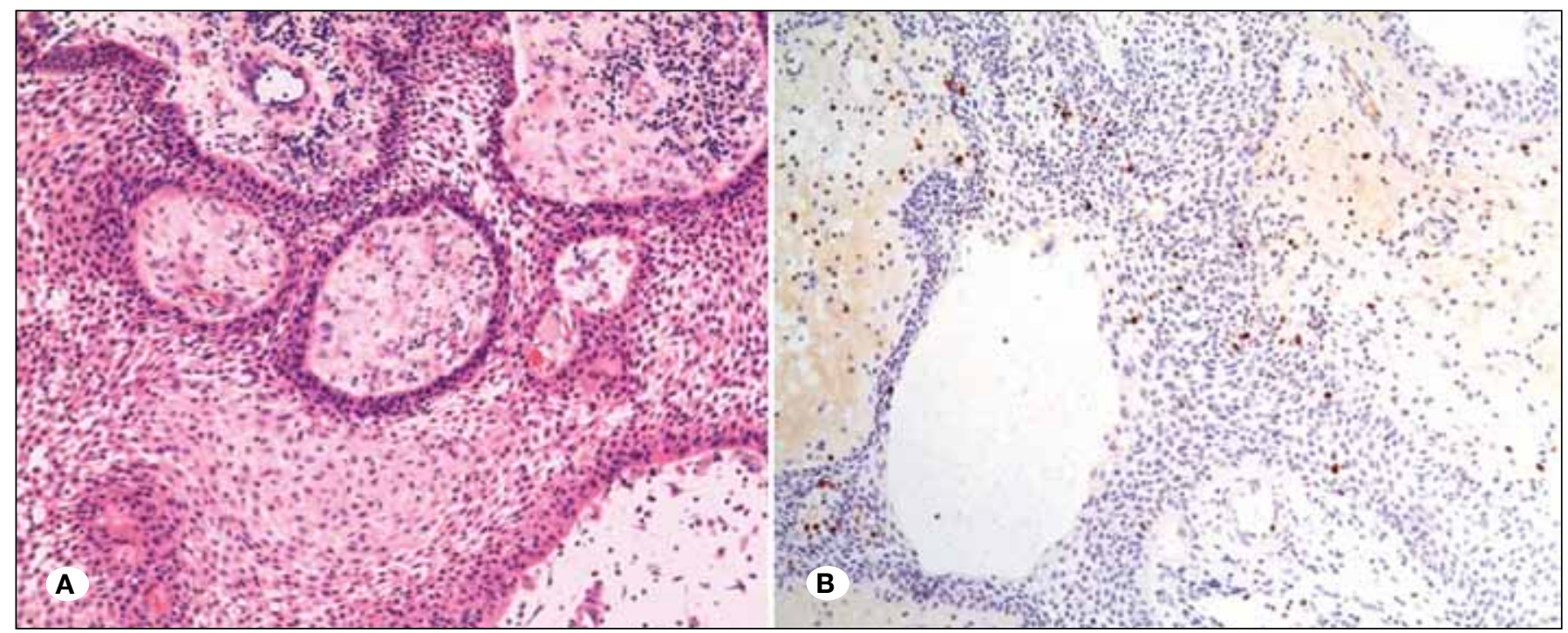

Figure 3: A) Hematoxylin\&Eosin staining of ectopic tumor confirm papillary craniopharyngioma; B) Ki-67 immunohistochemical staining showing proliferation index of $3 \%$.

dissemination are needed to elucidate the ectopic recurrence of craniopharyngioma. We suggest extra caution when operating on cystic craniopharyngioma. In addition, isolation of the tumor with cotton and complete aspiration of cystic fluid before resecting the capsule to avoid rupture of the cyst is vital. We have not been able to prevent these recurrences so far and long-term clinical and radiological follow-up of the patients is recommended.

\section{CONCLUSION}

Ectopic recurrence is rare incident following transcranial operation of craniopharyngioma. It seems to happen occasionally despite all efforts. Cystic tumors should be treated with extra caution to avoid dissemination to prevent ectopic recurrence to some degree. We conclude that GTR and long-term surveillance is the best way to treat craniopharyngioma. However, when prevention is not possible, complementary treatment with radiation therapy may be considered.

\section{REFERENCES}

1. Boongird A, Laothamatas J, Larbcharoensub N, Phudhichareonrat S: Malignant craniopharyngioma; case report and review of the literature. Neuropathology 29:591-596, 2009

2. Bunin GR, Surawicz TS, Witman PA, Preston-Martin S, Davis F, Bruner JM: The descriptive epidemiology of craniopharyngioma. J Neurosurg 89:547-551, 1998

3. Fahlbusch R, Honegger J, Paulus W, Huk W, Buchfelder M: Surgical treatment of craniopharyngiomas: Experience with 168 patients. J Neurosurg 90:237-250, 1999
4. Gao S, Shi X, Wang Y, Qian H, Liu C: Malignant transformation of craniopharyngioma: Case report and review of the literature. J Neurooncol 103:719-725, 2011

5. Garre ML, Cama A: Craniopharyngioma: Modern concepts in pathogenesis and treatment. Curr Opin Pediatr 19:471-479, 2007

6. Gupta DK, Ojha BK, Sarkar C, Mahapatra AK, Sharma BS, Mehta VS: Recurrence in pediatric craniopharyngiomas: Analysis of clinical and histological features. Childs Nerv Syst 22:50-55, 2006

7. Jakobs M, Orakcioglu B: Ectopic recurrent craniopharyngioma of the frontal bone. J Neurosurg 117:490-497, 2012

8. Louis DN, Ohgaki H, Wiestler OD, Cavenee WK, Burger PC, Jouvet A, Scheithauer BW, Kleihues P: The 2007 WHO classification of tumour of the central nervous system. Acta Neuropathol 114:97-109, 2007

9. Mortini P, Gagliardi F, Boari N, Losa M: Surgical strategies and modern therapeutic options in the treatment of craniopharyngiomas. Crit Rev Oncol Hematol 88:514-529, 2013

10. Nomura $H$, Kurimoto $M$, Nagai $S$, Hayashi $N$, Hirashima $\mathrm{Y}$, Tsukamoto E, Endo S: Multiple intracranial seeding of craniopharyngioma after repeated surgery-case report. Neurol Med Chir (Tokyo) 42:268-271, 2002

11. Ujifuku K, Matsuo T, Takeshita T, Hayashi K, Kitagawa N, Hayashi T, Suyana K, Nagata I: Malignant transformation of craniopharyngioma associated with moyamoya syndrome. Neurol Med Chir (Tokyo) 50(7):599-603, 2010

12. Zhao $\mathrm{X}$, Yi X, Wang $\mathrm{H}$, Zhao $\mathrm{H}$ : An analysis of related factors of surgical results for patients with craniopharyngiomas. Clin Neurol Neurosurg 114:149-155, 2012 